\title{
Morphologie und synaptische Interaktion von Neuronen einer kortikalen Kolumne
}

\author{
Joachim Lübke und Dirk Feldmeyer
}

\section{Zusammenfassung}

Ein grundlegendes Prinzip des Neokortex ist seine Organisation in funktionelle Kolumnen und ein weitreichendes horizontales System neuronaler Verbindungen. Diese Kolumnen mit ihren Netzwerken von Neuronen, die für alle sensorischen Hirnregionen nachgewiesen wurden, sind maßgeblich an der Generierung unterschiedlichster Wahrnehmungsprozesse unseres Gehirns beteiligt. Bisher ist jedoch relativ wenig darüber bekannt, wie komplexe Sinnesleistungen auf zellulärer Ebene verarbeitet werden.

In den letzten zehn Jahren wurden zahlreiche neuronale Verbindungen innerhalb und zwischen kortikalen Schichten funktionell und strukturell untersucht. Eine der wichtigsten Techniken ist dabei die simultane Patch-clamp Ableitung synaptisch gekoppelter Neurone bei gleichzeitiger intrazellulärer Biocytin-Füllung, die eine korrelierte Struktur-Funktionsanalyse erlaubt. Neben Gemeinsamkeiten zeigten sich jedoch groBe Unterschiede hinsichtlich der funktionellen und morphologischen Eigenschaften der synaptischen Transmission. Daraus folgt die Notwendigkeit, jede einzelne neuronale Verbindung separat zu beschreiben. Ziel dieser Untersuchungen ist letztlich, die Interaktion der einzelnen Verbindungen innerhalb des neuronalen Netzwerkes einer kortikalen Kolumne zu verstehen.

\begin{abstract}
Morphology and synaptic interaction between neurons in a cortical column

A basic feature of the neocortex is its organisation into functional columns and a longrange horizontal system of neuronal connections. These columns together with their network of neurones, present in all sensory cortices, are the cellular substrate for sensory perception in the brain. Currently, relatively little is known how these complex functions are realised at the level of individual neurones or networks.

In the last decade numerous neuronal connections within or between cortical layers have been studied both at a functional and structural level. Paired patch-clamp recordings from synaptically coupled neurones combined with simultaneous biocytinfillings is one of the most important techniques allowing a correlated structure-function analysis. Besides similarities, clear differences with respect to both physiology and morphology of synaptic transmission were found. Therefore it is essential to investigate each neuronal connection individually. The aim of these studies is to understand the interaction of individual neuronal connections within the framework of a cortical column.
\end{abstract}

Key words: cortical column, synaptic transmission, cortical signal flow, neuronal connectivity, synaptic efficacy

\section{Einleitung}

Ein generelles Organisationsprinzip sensorischer Areale des Neokortex ist ihre Gliederung in funktionelle vertikal orientierte Kolumnen. Das Konzept der kolumnären Organisation der Hirnrinde geht in erster Linie auf grundlegende Untersuchungen zur Repräsentation der Mechanorezeption im so genannten „Fässchen“- oder „Barrel“-Feld des somatosensorischen Kortex zurück (Mountcastle 1957; Mountcastle und Powell 1959). Ein zentraler Befund dieser Arbeiten war, dass alle Neurone einer vertikalen, durch alle kortikalen Schichten verlaufenden Kolumne nur durch eine der im somatosensorischen Kortex repräsentierten Sinnesmodalitäten (in diesem Fall: Schnurrhaarbe- wegung, Druckwahrnehmung der Haut und Tiefensensibilität) erregt werden. Diese klare Trennung einzelner sensorischer Modalitäten in alternierenden Kolumnen, in denen Neurone ähnliche Eigenschaften besitzen, führte zum Konzept der funktionellen kolumnären Organisation sensorischer Kortizes. Mit der Entdeckung von Orientierungs-, Richtungs- und okulären Dominanzkolumnen im visuellen Kortex durch Hubel und Wiesel $(1962,1963)$ wurde dieses Organisationsprinzip bestätigt und erweitert.

Neben den funktionellen vertikalen Kolumnen existiert ein diskontinuierliches, weitreichendes System von intra- bzw. translaminären neuronalen Verbindungen, welche zum einen verschiedene kortikale Areale, zum anderen die kortikalen Schichten miteinander verbinden. Dieses weitreichende System ist jedoch im Wesentlichen auf exzitatorische Neurone beschränkt. Diese Verbindungen scheinen bei der Synchronisation von räumlich nicht benachbarten, weit auseinander liegenden Ensembles von Pyramidenneuronen kortikaler Kolumnen mit ähnlicher Funktion, z.B. okulärer Dominanz und/oder ähnlicher Richtungs- bzw. Orientierungsspezifität, eine wichtige Rolle zu spielen.

Ein besonders attraktives System zum Studium neuronaler Netzwerke innerhalb einer kortikalen Kolumne ist der so genannte „Barrel“-Kortex von Nagetieren. Diese „Barrels“ stellen die topographisch exakte Repräsentation des Schnurrhaarsystems von Nagern im Neokortex dar. Dabei ist jedem Schnurrhaar ein definiertes „Barrel“ zugeordnet (Woolsey und van der Loos 1970). Im Vergleich zu anderen sensorischen Kortizes ist hier die Definition einer kortikalen Kolumne anhand struktureller Merkmale viel klarer, da die „Barrels“ schon im ungefärbten Präparat sichtbar sind (Abbildung 1C). Zudem ist die Zuordnung zum peripheren Rezeptor und zur sensorischen Modalität eindeutig, so dass eine klare Struktur-Funktionsbeziehung möglich ist.

Ein Weg, derartige Struktur-Funktionsbeziehungen zu untersuchen ist das akute Gehirnschnittpräparat. Ein grundlegender Vorteil gegenüber in vivo Experimenten im Ganztier ist hierbei der relativ einfache $\mathrm{Zu}$ gang zu den neuronalen Strukturen, so dass gezielte Messungen von identifizierten Neuronen unter optischer Kontrolle möglich sind (Abbildung 1A, B). Erste simultane Ableitungen von synaptisch gekoppelten Neuronen des Neokortex wurden von Thomson und Mitarbeitern (1994) publiziert. In dieser Arbeit wurden synaptische Verbindungen zwischen Pyramidenzellen der kortikalen 

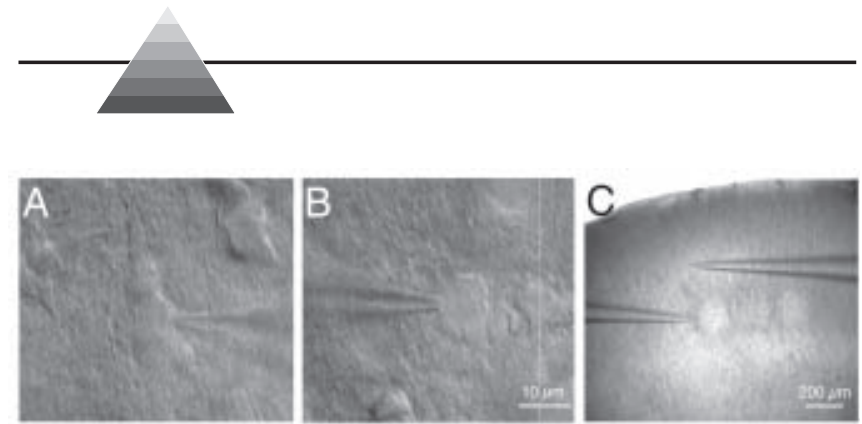

Abb. 1: Synaptisch gekoppelte, exzitatorische Neurone in Schicht 4 und Schicht 2/3 des „Barrel“-Kortex. Infrarot-Differenzialinterferenzkontrast (IR-DIC)-Aufnahmen einer postsynaptischen Pyramidenzelle in Schicht 2/3 (A) und einer präsynaptischen Sternzelle in Schicht 4 (B) bei hoher Mikroskopvergrößerung. Die beiden Elektroden in (C) zeigen die relative Lage des prä- und postsynaptischen Neurons zueinander. Im ungefärbten Gehirnschnittpräparat sind drei benachbarte „Barrels“ gut zu erkennen, die durch Septa eindeutig voneinander getrennt sind. Die beiden synaptisch gekoppelten Neurone liegen direkt in bzw. oberhalb eines kortikalen „Barrels“, d.h. beide Neurone sind in derselben kortikalen Kolumne lokalisiert.

Schichten 5 und 6 abgeleitet und anschließend morphologisch dargestellt. Dadurch konnte eine Beziehung zwischen der Amplitude des exzitatorischen postsynaptischen Potentials und der Anzahl der synaptischen Kontakte hergestellt werden; d.h. hier wurden erstmals funktionelle Eigenschaften synaptischer Transmission mit morphologischen Parametern korreliert. Leider waren von über 1000 gemessenen Neuronenpaaren nur 35 tatsächlich miteinander verbunden, und nur bei drei Verbindungen war ein qualitativ ausreichender Strukturerhalt gegeben, so dass viele wichtige Aspekte synaptischer Transmission weiterhin ungeklärt blieben.

Ein Durchbruch waren gezielte simultane Patch-clamp Ableitungen von identifizierten Pyramidenzellen der Schicht 5 (Markram et al. 1997). Dieser experimentelle Ansatz ermöglicht, gezielte Fragen zu funktionellen und strukturellen Parametern synaptischer Transmission zu stellen: Welche Rolle spielen funktionelle Faktoren wie die Größe und der Zeitverlauf der postsynaptischen Leitfähigkeitsänderung bei der Effizienz der synaptischen Übertragung und wie beeinflussen morphologische Faktoren wie die dendritische Konfiguration, axonale Projektion sowie die Anzahl und Verteilung synaptischer Kontakte zusammen mit den aktiven und passiven elektrophysiologischen Eigenschaften des prä- und postsynaptischen Neurons die Effizienz synaptischer Übertragung?

Individuelle synaptische Verbindungen wurden dann systematisch durch Verwendung des „Barrel“-Kortex-Gehirnschnittpräparats in Bezug auf das neuronale Netzwerk einer kortikalen Kolumne untersucht (Feldmeyer et al. 1999). Hier wurde der Versuch unternommen, die Konnektivität oder Verbindungsrate am Beispiel von exzitatorischen Neuronen der Schicht 4 abzuschätzen und einen Bezug zur kolumnären Struktur herzustellen (Lübke et al. 2000; s.a. Abbildung 1,3). Dadurch wurde erstmals ein Brückenschlag zwischen der zellulären Beschreibung neuronaler Verbindungen und der Systemebene erreicht.

In den folgenden Kapiteln wird der heutige Kenntnisstand zur Morphologie und Physiologie synaptischer Transmission im Neokortex anhand der wichtigsten Parameter zusammengefasst und basierend auf diesen Erkenntnissen versucht, den Signalfluss in einer kortikalen Kolumne darzustellen.

\section{Konnektivität in einer kortikalen Kolumne}

Lange Zeit wurde angenommen, dass die Konnektivität im Neokortex eher zufällig ist (Braitenberg und Schüz 1998). Dagegen sprechen neuere Untersuchungen, die eindeutig belegen, dass es mehre-

Neuroforum 3/04

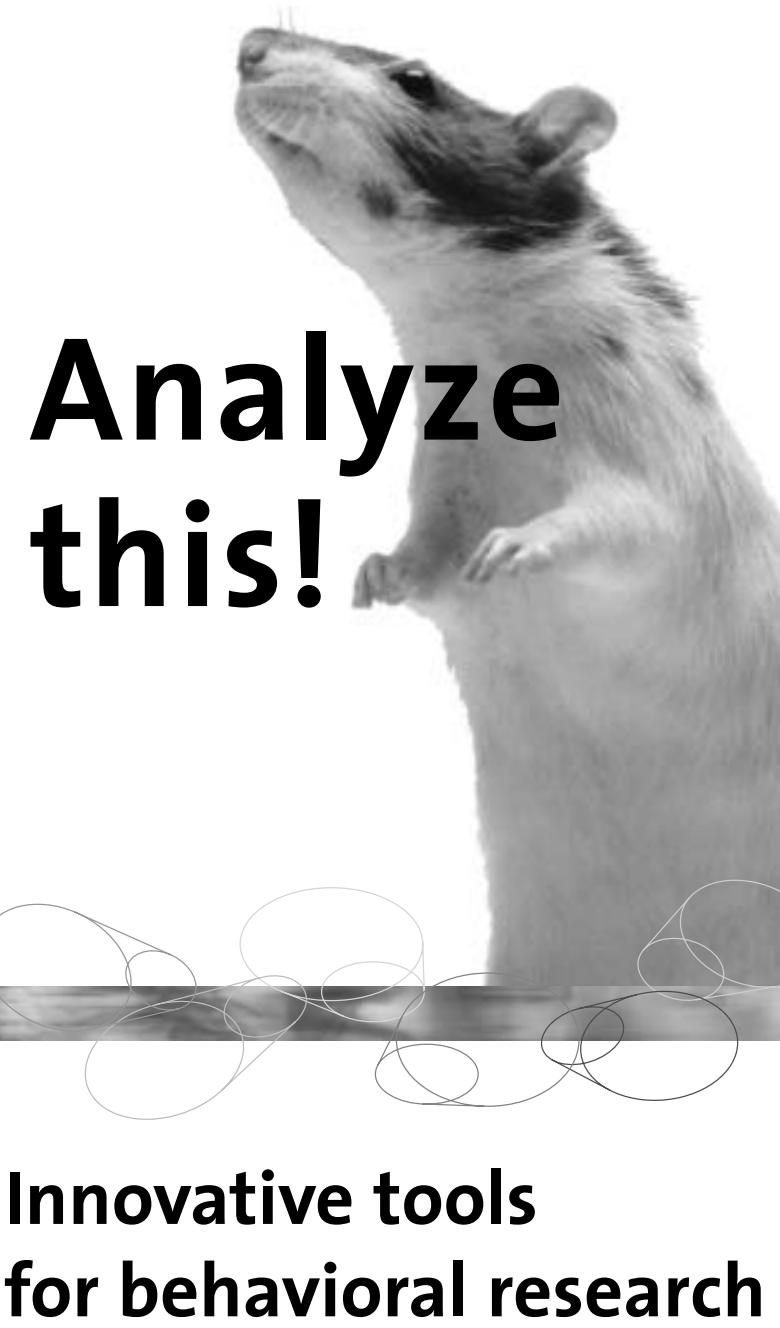

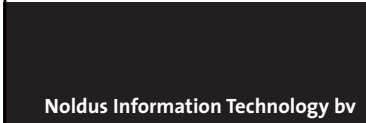

Wageningen, The Netherlands

Phone: $\quad+31-317-497677$

E-mail: info@noldus.nl

Noldus Information Technology GmbH

Freiburg, Germany

Phone: $\quad+49-761-4701600$

E-mail: info@noldus.de

Noldus Information Technology Inc.

Leesburg, VA, U.S.A.

Phone: +1-703-771-0440

Toll-free: 1-800-355-9541

E-mail: info@noldus.com

www.noldus.com
Scientists studying animal behavior

have an increasing need for accurate quantitative data. As a behavioral neuroscientist, you need sensitive observational research tools with a maximum degree of automation. Our integrated solutions for data collection, analysis, management and visualization are today's premier tools for the study of behavior, locomotion and acoustics.

EthoVision - Video tracking system for automation of behavioral experiments The Observer - System for collection and analysis of observational data, live or from video UltraVox - System for automatic monitoring of ultrasonic vocalizations 


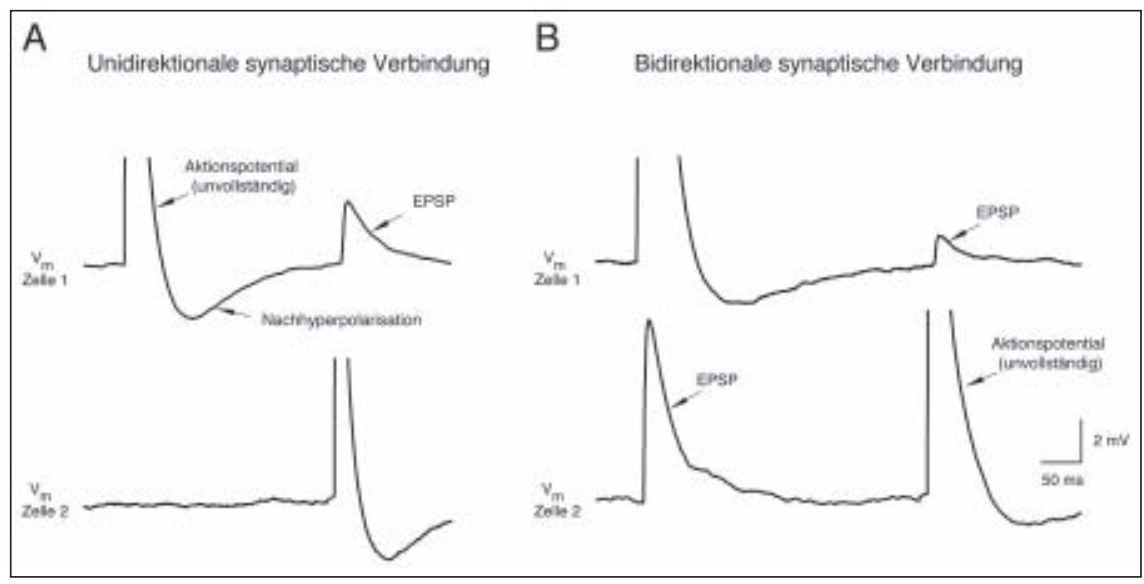

Abb. 2: Uni- und bidirektionale (reziproke) synaptische Verbindung. (A) Ein Aktionspotential in Zelle 1 löst kein exzitatorisches postsynaptisches Potential (EPSP) in Zelle 2 aus; umgekehrt löst ein Aktionspotential in Zelle 2 ein EPSP in Zelle 1 aus; die Verbindung ist demnach uni-direktional. (B) Aktionspotentiale in Zelle 1 als auch in Zelle 2 lösen EPSPs in Zelle 2 bzw. Zelle 1 aus; d.h. die Verbindung ist bidirektional; in derartigen Verbindungen kommt es zu rekurrenter Erregung.

re Grundprinzipien der kortikalen Organisation und neuronalen Verschaltung gibt. Qualitativ kann zwischen lokalen, intralaminären Verbindungen (innerhalb einer kortikalen Schicht) und translaminären (zwischen einzelnen kortikalen Schichten) bzw. transkolumnären (zwischen kortikalen Kolumnen) unterschieden werden. Darüber hinaus ist die synaptische Verschaltung entweder uni-direktional oder reziprok (bidirektional; Abbildung 2). Während die uni-direktionale Verschaltung einen einseitig gerichteten Signalfluss bedingt, ermöglicht die bidirektionale Kopplung eine rekurrente Erregung bzw. eine Rückkopplungsinhibition (Feed-back inhibition).

Die überwiegende Zahl der bisher untersuchten synaptischen Verbindungen des Neokortex ist lokal (d.h. innerhalb einer Distanz von $<150 \mu \mathrm{m}$ ) und intralaminar. Dies gilt für exzitatorische, aber mehr noch für inhibitorische synaptische Verbindungen. Ein hoher Prozentsatz der intralaminären Verbindungen ist reziproker Natur. Nach heutigem Kenntnisstand sind translaminäre Verbindungen dagegen uni-direktional (Thomson 1997; Reyes und Sakmann 1999; Tarczy-Hornoch et al. 1999; Feldmeyer et al. 2002). Die synaptische Signaltransduktion ist ebenfalls gerichtet und zwar im Wesentlichen von den sensorischen Relay-Kernen des Thalamus nach Schicht 4 des Neokortex, der ersten Station kortikaler Signalverarbeitung (Abbildung 3, 4). Von dort wird die synaptische Erregung über exzitatorische Neurone der kortikalen Schicht 4 zunächst auf Pyramidenzellen der Schicht 2/3 und dann auf solche der Schicht 5 übertragen, d.h. der Informationsfluss ist strikt uni-direktional. In Übereinstimmung hiermit finden sich synaptische Verbindungen zwischen Schicht 4 und Schicht 2/3 (Feldmeyer et al. 2002) bzw. Schicht $2 / 3$ und Schicht 5 häufig (Thomson 1997; Reyes und Sakmann 1999). Synaptische Verbindungen von Schicht 5 nach Schicht 2/3 sind dagegen bis heute nicht nachgewiesen worden.

Auf zellulärer Ebene spielt die Zielspezifität der Axone zur Beschreibung der Konnektivität individueller neokortikaler Verbindungen eine wesentliche Rolle. Dabei kommt sowohl zelluläre (d.h. neuronenklassenspezifische) wie auch subzelluläre Zielspezifität vor, d.h. synaptische Kontakte terminieren an einem bestimmten neuronalen Kompartiment, entweder am Soma, den Dendriten oder am Axonhügel. Für die verschiedenen Klassen GABAerger Interneurone ist eine derartige Zielspezifität seit langem bekannt; Untersuchungen an exzitatorischen synaptischen Verbindungen haben jedoch gezeigt, dass auch hier eine gerichtete Termination axonaler Boutons (Verdickungen des Axons im Bereich eines synaptischen Kontakts) vorliegt.

Kartierungen zur räumlichen Verteilung synaptischer Verbindungen von Pyramidenneuronen der kortikalen Schicht 5 mittels Kombination von Patch-clamp Ableitungen und durch Photolyse freigesetztes Glutamat aus einer licht-sensitiven inaktiven Vorstufe (engl. caged-compounds) haben gezeigt, dass offensichtlich Subpopulationen von Pyramidenneuronen in der Schicht 5 existieren. Diese weisen in Bezug auf ihre synaptischen Eingänge schichtenspezifische Unterschiede auf (Schubert et al. 2001). Leider kann mit diesem Versuchsansatz der Ursprung dieser Inputs (d.h. die Projektionsneurone), welche zur synaptischen Antwort beitragen, nur unzureichend zurückverfolgt werden.
Quantitativ wird die Konnektivität eines gegebenen Netzwerks durch die Häufigkeit der synaptischen Kopplung zwischen verschiedenen Neuronentypen bestimmt. Dabei sind allerdings folgende Punkte kritisch zu betrachten und bei der Berechnung von Konnektivitätsraten zu berücksichtigen: (1) Die experimentelle Wahrscheinlichkeit bzw. der Bias (Erfahrung, Selektion häufig vorkommender Neuronentypen usw.), im akuten Gehirnschnitt eine Verbindung zu finden; (2) Die Wahrscheinlichkeit synaptisch gekoppelter Neurone in akuten Gehirnschnitten stimmt nicht mit dem tatsächlichen Grad der Konnektivität in vivo überein; (3) Synaptisch gekoppelte Neurone zeigen ein weites Spektrum unterschiedlicher Morphologien bezüglich ihres axonalen Projektionsmusters, der Dichte und Verteilung axonaler Boutons und ihrer Anordnung (z.B. in Form neuronaler Cluster) innerhalb einer Hirnregion oder in einer kortikalen Kolumne.

Der Grad der Konnektivität einer Verbindung wurde zuerst an Hand der experimentellen Erfolgsrate, eine synaptische Verbindung zu finden, bestimmt. Dies ist jedoch kein allzu verlässlicher Parameter, da je nach experimentellen Bedingungen die Konnektivitätsrate starken Schwankungen unterliegt, wie das Beispiel exzitatorischer Schicht 5-Verbindungen verdeutlicht $(1.4 \%$, Thomson et al. 1993; 15\%, Markram et al. 1997; 10\%, Sjöström et al. 2001). Die experimentelle Erfolgsrate kann daher nur als ein grobes $\mathrm{Ma} \beta$ zur Beschreibung der Konnektivität einer Verbindung herangezogen werden. Um zu realistischen Angaben zu kommen, müssen also Parameter wie die räumliche Verteilung und Dichte synaptischer Boutons, die mittlere Anzahl synaptischer Kontakte pro Verbindung und die Anzahl von Neuronen, die innerhalb des rezeptiven Feldes des präsynaptischen Axons liegen, für die Berechnung der Konnektivität mit einbezogen werden. Bis heute liegen solche Analysen nur für intralaminäre Verbindungen zwischen Schicht 5-Pyramidenzellen (15-25\%, Markram 1997) und exzitatorischen Neuronen der Schicht 4 vor (20\%, Feldmeyer et al. 1999). Aber auch hier bleibt unberücksichtigt, dass die Konnektivitätsrate mit steigender Distanz der prä- und postsynaptischen Neurone (intra- vs. transkolumnäre Verbindungen) deutlich abnimmt und stark von der unterschiedlichen Dichte und Verteilung synaptisch gekoppelter Neurone in den einzelnen kortikalen Schichten (Feldmeyer et al. 1999, 2000) und von der Zielspezifität einzelner Neurone einer gegebenen Verbindung beeinflusst wird (Lübke et al. 2000, 2003). Diese Abhängigkeit wird besonders deutlich, weil z.B. der Anteil reziproker 


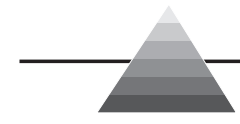

Verbindungen ( $30 \%$ für intralaminäre Verbindungen) die berechnete Konnektivitätsrate deutlich übersteigt. Reziproke kortikale Verbindungen werden als Feed-back -Verstärker synaptischer Signale angesehen, obwohl der Nettoeffekt der Verstärkung kritisch von der synaptischen Effizienz, der Feed-forward-Hemmmung und dem Vorkommen bzw. der Dichte solcher Verbindungen abhängt (Douglas et al. 1995; Chance et al. 1999).

Ein weiterer wichtiger Parameter zur Beschreibung der Konnektivität ist die Anzahl und Verteilung synaptischer Kontakte am postsynaptischen Neuron. Die Zielspezifität synaptischer Kontakte GABAerger Interneurone auf Prinzipalneurone oder andere GABAerge Interneurone ist im Gegensatz zu rein exzitatorischen Verbindungen relativ gut untersucht (Kawaguchi und Kubota 1997, 1998; Tamás et al. 1997, 1998; Finnerty et al. 1999; Galarreta und Hestrin 1999, 2001; Gupta et al. 2000; Krimer und Goldmann-Rakic 2001; Kaiser et al. 2004). Dabei etablieren die Axone GABAerger Interneurone en passant Boutons an spezifischen Kompartimenten des Zielneurons, d.h. entweder am Soma, an den Dendriten oder direkt am Axoninitialsegment, welches den unterschiedlichen Einfluss der Hemmung auf die postsynaptische Partnerzelle (selektive Unterdrückung synaptischer Inputs an unterschiedlichen Kompartimenten des Zielneurons, Blockade der Initiierung von $\mathrm{Na}^{+}$und $\mathrm{Ca}^{2+}$ Spikes usw.) und damit auch innerhalb eines Netzwerks erklärt.

Neuere Studien zu synaptischen Verbindungen zwischen exzitatorischen Neuronen des Neokortex zeigen eindeutig, dass sich solche Verbindungen auch durch eine hohe Zielspezifität auszeichnen. In allen bisher untersuchten Verbindungen sind synaptische Kontakte exklusiv nur am Dendritenschaft bzw. an dendritischen Dornenfortsätzen (engl. spines) zu finden, und dort hauptsächlich an sekundären und tertiären basalen Dendriten (63-85\%) lokalisiert (Makram et al. 1997a; Feldmeyer et al. 1999, 2002; Lübke et al. 2000, 2003; Silver et al. 2003). Die große Mehrzahl synaptischer Kontakte befindet sich zudem relativ nah am Soma $(\sim 100 \mu \mathrm{m})$ und trägt so auf Grund der geringen elektrotonischen Distanz zur synaptischen Effizienz bei, da die elektrische Filterung des postsynaptischen Potentials nicht sehr stark ist.

Interessanterweise scheint es Ausnahmen zur Verteilung synaptischer Kontakte einer exzitatorischen Verbindung zu geben. Die bisher beschriebenen Verbindungen sind alle durch eine synaptische Innervationsdomäne gekennzeichnet, die sich hauptsächlich im Bereich der basalen Dendriten des postsynaptischen Neurons befindet. Für die Verbindungen zwischen Pyramidenzellen der Schicht 2/3 und der Schicht 5 sind synaptische Kontakte nicht nur an basalen Dendriten lokalisiert, sondern auch in der Nähe der Endverzweigung des apikalen Dendriten. Dies ist die Region, an der dendritische Kalzium-Aktionspotentiale ausgelöst werden, so dass hier EPSPs zu deren Auslösung beitragen können.

\section{Signalfluss in einer kortikalen Kolumne (Kanonischer Signalfluss)}

Der Signalfluss bzw. die Signalverarbeitung in einer kortikalen Kolumne ist für die exzitatorische Transmission mittlerweile recht gut untersucht. Neben funktionellen Eigenschaften wie Konnektivität, Effizienz und Verlässlichkeit synaptischer Verbindungen bestimmen strukturelle Charakteristika wie die dendritische Konfiguration, axonale Projektion sowie die Anzahl und Verteilung morphologisch identifizierter synaptischer Kontakte den Signalfluss bzw. Signalverarbeitung einer kortikalen Kolumne.

Sensorische Signale aus der Peripherie erreichen über die spezifischen Relay-Kerne des Thalamus die Eingangsstation des Neokortex,

Neuroforum 3/04

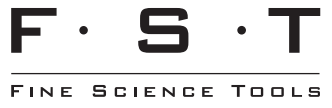

\section{Fine surgical instruments and accessories for research}

- Spring scissors

- Forceps

- Scalpels

- Sutures

- Retractors

- Clamps

- And much more

Fine Science Tools GmbH

Fahrtgasse 7 - 13

D-69117 Heidelberg

Germany

Tel.: +49 (0) $6221 / 905050$

Fax: +49 (0) $6221 / 600001$

E-Mail: europe@finescience.com

Web: www.finescience.com

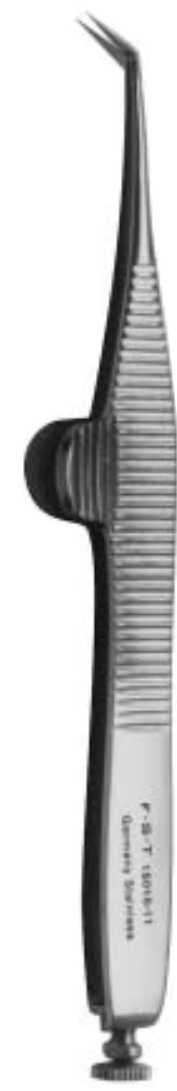

\section{Glaskapillaren zur Herstellung von}

Mikroelektroden, Mikropipetten, Patch-Pipetten, etc.

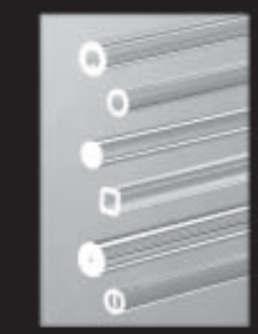

In allen Längen lieferbar...
Direkt vom Hersteller: glas for
wissonschatt
labor
industrie
technik

auch mit feurpolierten Enden ...

... aus Borosilicat-, Soda-, Quarz- und Bleiglas

Verschiedene Formen:

- rund, eckig

- multibarrel

- Theta, Filament

- 2-Loch, 4-Loch

- usw.

\section{- super dünn \\ - dünn \\ - normal \\ - dick \\ - sehr dick}

Verschiedene Wandstärken:

Neu:

Spezialnadeln ab $70 \mu \mathrm{m}$ Durchmesser,

zum Befüllen von Mikroelektroden und

Mikropipetten!

Hilgenberg GmbH, Strauchgraben 2, D - 34323 Malsfeld

Tel. ++49 (0) 566173030 Fax ++49 (0) 5661730311

Email info@hilgenberg-gmbh.de Internet: www.hilgenberg-gmbh.de 


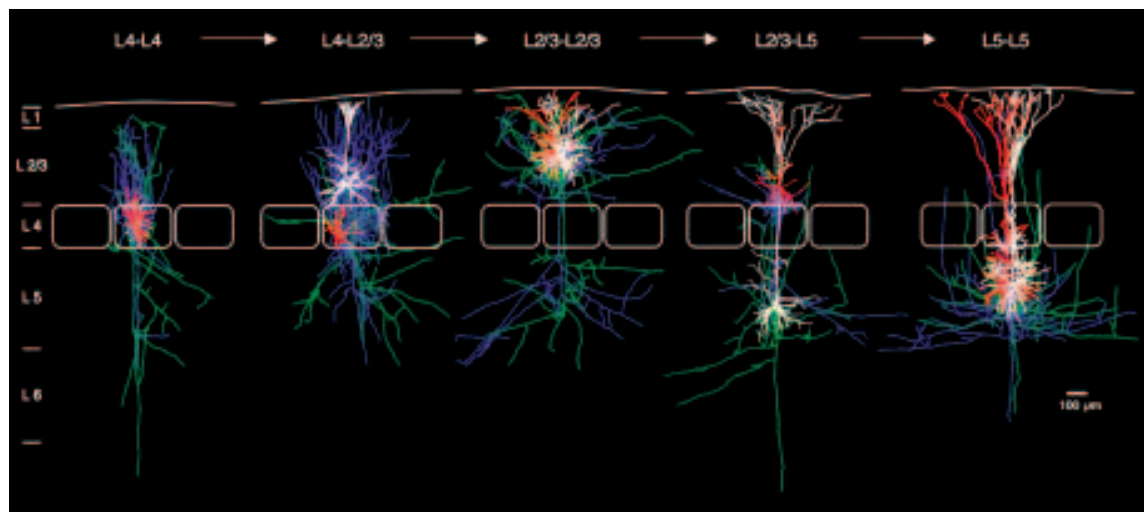

Abb. 3: Synaptische Verbindungen im Signalfluss einer kortikalen Kolumne. NEUROLUCIDA Rekonstruktionen synaptisch verbundener Paare exzitatorischer Neurone in der Signalkette einer kortikalen Kolumne. Die Dendriten der präsynaptischen Neurone sind rot dargestellt und ihre Axone in blau. Die postsynaptischen Neurone sind in weiß wiedergegeben und ihre Axone in grün. Der Signalfluss ist so dargestellt wie in Abbildung 4 detailliert beschrieben.

die Schicht 4 (Abbildung 3, 4). Die thalamokortikalen Afferenzen terminieren zum großen Teil an exzitatorischen Sternzellen, d.h. diese Zellen repräsentieren die erste Station kortikaler Signalverarbeitung. Für den visuellen und den somatosensorischen Kortex konnte gezeigt werden, dass synaptische Verbindungen dieser Neurone durch eine hohe Effizienz gekennzeichnet sind, welche unter anderem durch die Höhe der mittleren EPSP-Amplitude (1.0-1.6 mV) und der geringen Fehlerrate $(<5 \%)$ bzw. dem niedrigen Koeffizienten der Variation (CV < 0.4; Quotient aus der Standardabweichung und dem Mittelwert der EPSP-Amplitude; er gibt die relative Varianz des mittleren Messwertes an) ausgedrückt wird (Stratford et al. 1996; Tarczy-Hornoch et al. 1999; Feldmeyer et al. 2002). Einige dieser Schicht 4-Verbindungen sind so stark, dass es zur Auslösung postsynaptischer Aktionspotentiale kommt, die wahrscheinlich auf die vergleichsweise hohe Beteiligung von NMDA-Rezeptoren $(\sim 40 \%)$ an der Gesamtamplitude zurückzuführen sind (Feldmeyer et al. 1999). Auf Grund der hohen Konnektivität zwischen exzitatorischen Sternzellen dieser Schicht (Feldmeyer et al. 1999; Lübke et al. 2003) kommt diesen Neuronen nicht nur eine Schlüsselstellung bei der Signalübertragung, sondern auch eine „Feed-back-Verstärker“" Funktion selbst schwacher sensorischer Signale aus der Peripherie zu (Douglas et al. 1995).

Morphologisch sind exzitatorische Sternzellen durch die vertikale, auf die Kolumne beschränkte, Projektion ihres Axons charakterisiert (Abbildung 3, 4). Diese Axonkollateralen ziehen dabei durch alle kortikalen Schichten mit einer präferentiellen Projektion in Schicht 2/3, wo die en passant Bou- tons zielspezifisch an Pyramidenneuronen zu finden sind. Der Signalfluss von Schicht 4 nach Schicht 2/3 (translaminar) ist uni-direktional, d.h. nur in Richtung der Schicht 2/3 und nicht umgekehrt und bleibt auf die Kolumne beschränkt (Feldmeyer et al. 2002; Lübke et al. 2003; s. a. Abbildung 3, 4).

Obwohl die Distanz zwischen prä- und postsynaptischem Neuron nicht unerheblich ist, ist diese synaptische Verbindung trotz ihrer relativ niedrigen Effizienz (mittlere EPSP-Amplitude $0.7 \mathrm{mV}$ ) sehr zuverlässig. Für die Signalübertragung von Schicht 4 nach Schicht 2/3 scheint daher eine wesentdie gleichzeitige Rekrutierung vieler Sternzellen notwendig zu sein. Pyramidenneuronen der Schicht 2/3 kommt folglich eher eine Filterfunktion für die laterale Ausbreitung der Erregung innerhalb dieser Schicht zu.

Im Gegensatz zur kolumnären Organisation der Axone der exzitatorischen Schicht 4-Neurone verfügen Schicht 2/3-Pyramidenneurone über zwei axonale Domänen: eine vertikale, hauptsächlich kolumnär organisierte Domäne und ein weitreichendes diskontinuierliches System horizontaler Verbindungen präferentiell in den Schichten 2/3 und 5, mit einer Projektion individueller Axonkollateralen von 2-4 mm über die Kortexoberfläche (Abbildung 3).

An dieser Stelle des Neokortex kann der Signalfluss zwei Richtungen nehmen, entweder innerhalb der Kolumne (intrakolumnär) oder über Kolumnen hinweg (transkolumnär). Die Zielstruktur von Schicht 2/3Pyramidenzellaxonen sind hauptsächlich andere Schicht 2/3- oder Schicht 5-Pyramidenneurone. Für synaptische Verbindungen (Paarableitungen) zwischen Pyramidenneuronen der Schicht 2/3 liegen bis heute hauptlich höhere Schwelle der Aktivierung bzw. sächlich Befunde zur lokalen (Distanz zwischen prä- und postsynaptischen Neuron $\sim 50 \mu \mathrm{m}$ ), intrakolumnären (Reyes und Sakmann 1999; Atzori et al. 2001; Feldmeyer et al. 2003; Thomson und Bannister 1998, 2003) und nur eine Arbeit zur transkolumnären Konnektivität (Yoshimura et al. 2000) vor. Der Signalfluss innerhalb von Schicht 2/3 ist sowohl uni- (Atzori et al. 2001) als auch bidirektional und wird über die vertikale und horizontale axonale Domäne vermittelt.

Obwohl die Konnektivität von Pyramidenneuronen der Schicht 2/3 lokal (Radius von $\sim 100 \mu \mathrm{m})$ sehr hoch ist, ist die Effizienz dieser Verbindung vergleichsweise niedrig (mittlere EPSP Amplitude 0.3-1.0 mV). Auch die Wahrscheinlichkeit der Neurotransmitter-Freisetzung (probability of release, kurz $P_{r}$ ) und die synaptischen Antworten nach repetitiver Stimulation sind bei diesen Verbindungen in verschiedenen sensorischen Kortizes sehr unterschiedlich. Während im auditorischen Kortex zwei Subpopulationen synaptischer Schicht 2/3-Pyramidenzell-Verbindungen, eine mit niedrigem $P_{r}(0.13)$ und eine zweite mit hohem $P_{r}(0.68)$ vorkommen, existieren im Barrel-Kortex nur solche mit hohem $P_{r}(0.93)$. Synaptische Verbindungen zwischen Neuronen gleichen Typs aber unterschiedlichen physiologischen Eigenschaften sind auch für den motorischen, somatosensorischen und visuellen Kortex nachgewiesen (Thomson 1997).

Diese Befunde implizieren, dass sowohl innerhalb eines sensorischen Areals als auch zwischen sensorischen Kortizes sehr heterogene Verbindungstypen zwischen Pyramidenzellen der kortikalen Schicht 2/3 existieren. Die Signaltransduktion über diese Verbindungen ist daher in verschiedenen sensorischen Arealen sehr unterschiedlich und wahrscheinlich dem jeweiligen sensorischen Input angepasst. Zudem repräsentieren Schicht 2/3-Pyramidenzellen eine sehr heterogene Population innerhalb der Klasse der Pyramidenneurone hinsichtlich ihrer dendritischen Geometrie und axonalen Projektion, die möglicherweise das morphologische Substrat für die Unterschiede in funktionellen Eigenschaften darstellen. Intralaminäre Schicht 2/3-Pyramidenzellverbindungen sind integrative Elemente der Signalverarbeitung innerhalb einer kortikalen Kolumne. Über ihr diskontinuierliches System weitreichender horizontaler Axone findet die transkolumnäre Signaltransduktion statt. Eine mögliche Funktion könnte daher in der Synchronisation elektrischer Aktivität neuronaler Ensembles mit ähnlichen funktionellen Eigenschaften über einen großen Bereich 
der Kortexoberfläche, d.h. über mehrere kortikale Kolumnen hinweg, liegen.

Die andere Zielstruktur von Schicht 2/3Pyramidenzellaxonen sind Pyramidenzellen der Schicht 5, die in der Regel eine große terminale dendritische Endaufzweigung in Schicht 1 ausbilden. Die Pyramidenzellen der Schicht 5 stellen neben denen der Schicht 6 das „Output“"-System des Neokortex dar, z.B. in verschiedene subkortikale Gehirnregionen (z.B. Thalamus, Pons) bis hin zur sensorischen Peripherie (Abbildung 3, 4).

Translaminäre Verbindungen zwischen Pyramidenneuronen der Schicht 2/3 und Schicht 5 sind wahrscheinlich uni-direktional, wie die zwischen Schicht 4 nach Schicht 2/3 (Thomson 1997; Thomson und Bannister 2003; Reyes und Sakmann 1999). Die mittlere EPSP Amplitude variiert stark (0.1-0.8 mV), zudem scheint diese Verbindung eine gemessen an anderen Verbindungen relativ niedrige synaptische Effizienz zu haben. Ihre funktionelle Bedeutung ist relativ unklar, es ist jedoch vorstellbar, dass sie eine intrakolumnäre Relay-Station zur Integration kortikaler Signale von intra- und transkolumnären neuronalen Ensembles darstellt.

Neben Pyramidenzellen der Schicht 6 repräsentieren die der Schicht 5 den kortikalen Output zu subkortikalen Gehirnregionen bzw. zur sensorischen Peripherie und sind daher strukturell und funktionell am besten charakterisiert (Thomson et al. 1993; Deuchars et al. 1994; Markram und Tsodyks 1996; Markram et al. 1997a, b; Tsodyks und
Abb. 4: Signalfluss in einer kortikalen Kolumne. Die Abbildung zeigt schematisch den „kanonischen“ Signalfluss in einer kortikalen Kolumne eines sensorischen Areals des Großhirns. Als Beispiel wurde der somatosensorische „Fässchen“- oder „Barrel"-Kortex der Nager gewählt, weil hier in der kortikalen Schicht 4 die morphologischen Korrelate der kortikalen Kolumne, die „Barrels“ zu sehen sind (s. Text). Über thalamische Relay-Kerne erhält der Kortex sensorische Eingänge aus der Peripherie, die hauptsächlich in Schicht 4 terminieren. Exzitatorische Neurone der Schicht 4 verstärken das Signal und leiten es präferentiell an Pyramidenzellen der Schicht 2/3 weiter. Von dort wird es auf andere Pyramidenzellen in Schicht 2/3 oder auch auf solche in Schicht 5 übertragen. Pyramidenzellen in Schicht $\mathbf{5}$ sind ebenfalls stark reziprok untereinander verbunden und sind die Ausgangsstation des sensorischen Kortex; von hier gelangt das sensorische Signal in andere Kortexareale und subkortikale Bereiche.

Markram 1997). Die Verbindung von Pyramidenzellen der kortikalen Schicht 5 zeichnet sich durch eine relativ hohe Effizienz der synaptischen Transmisson aus, was sich in einer hohen mittleren EPSP Amplitude (1.3 $\mathrm{mV}$ ), einem niedrigen $\mathrm{CV}$ von 0.52 und einer geringen Fehlerrate (14\%) ausdrückt. Ensembles von synaptisch gekoppelten Schicht 5-Pyramidenneuronen scheinen sowohl intra- als auch transkolumnär bei de

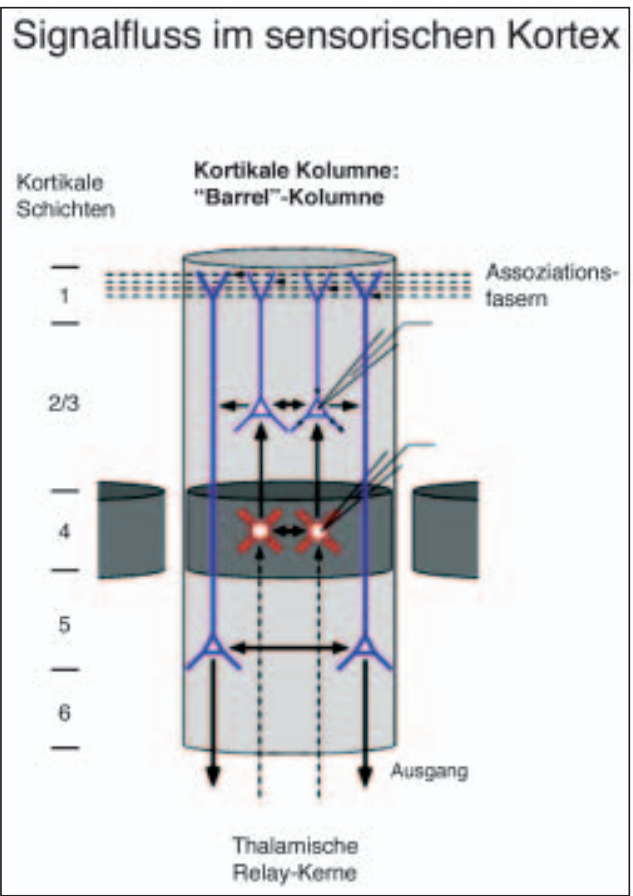

Generierung, Synchronisation und Aufrechterhaltung elektrischer Aktivität ein wichtiges Element der Signaltransduktion bzw. Signalverarbeitung darzustellen.

Im visuellen Kortex kommt eine zusätzliche Verbindung zwischen exzitatorischen Schicht 4-Neuronen und Schicht 6-Pyramidenneuronen vor (Tarczy-Hornoch et al. 1999). Interessanterweise weicht diese Verbindung in vielerlei Hinsicht von den oben

\section{SCIENCE PRODUCTS offers the newest $C E D$ soft- and hardware}

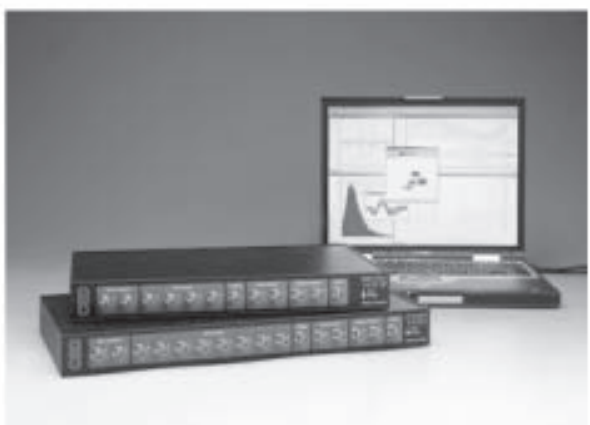

Hardware:

- The micro 1401 mk II with USB 2.0. A low- cost versitale data acquisition unit.

- The power 1401 with USB 2.0. CED's latest high preformance data acquisition interface. ( most powerfull life science laboratory interface in the world).
Science Products $\mathrm{GmbH}$ has offered CED Data Acquisition and Analysis Systems for over 20 years, in Germany, Austria and Switzerland.

The CED soft- and hardware already used in thousends of laboratories world- wide for a broad range of applications.

Software requires one of the CED 1401 series of intelligent laboratory interfaces and $a$ PC with Windows 98, Me,NT 4. Windows 2000 or XP and $a$ minimum of $256 \mathrm{MB}$ RAM.

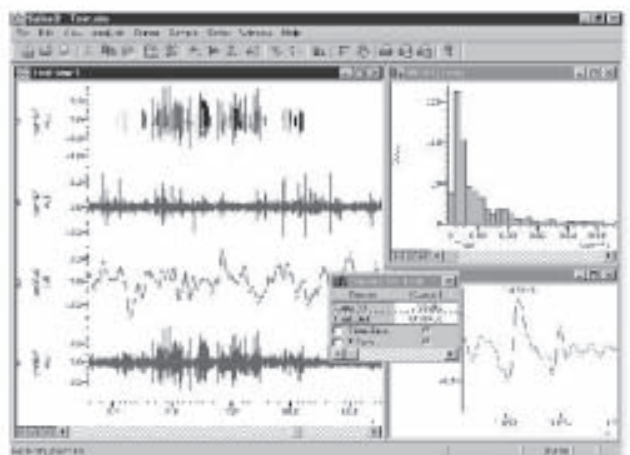

Software:

- Spike 2 Version 5 delivers powerfull data capture and analysis, stimulus sequencing and experimental control

- Signal Version 3 delivers powerfull sweep- based data capture and analysis, stimulus generation and control using one of the CED 1401 data acquisiton periphals. 
beschriebenen exzitatorischen Verbindungen $\mathrm{ab}$. Sie zeichnet sich durch eine vergleichsweise sehr niedrige synaptische Effizienz aus, hat einen niedrigen $P_{r}(0.13)$ und zeigt eine im Gegensatz zu anderen exzitatorischen Verbindungen frequenzabhängige Faszilitierung der EPSP Amplitude.

Obwohl bis heute die Vielzahl möglicher synaptischer Verbindungen des kolumnären Netzwerks des Neokortex noch nicht ausreichend bzw. gar nicht beschrieben ist, kann zusammenfassend festgestellt werden, dass intralaminäre Verbindungen in der Regel durch eine hohe synaptische Effizienz, eine geringe Fehlerrate und einen hohen Anteil reziproker Verbindungen charakterisiert sind. Im Gegensatz dazu sind translaminäre Verbindungen ein weitgehend kolumnäres, uni-direktionales System, welches den gerichteten Informationsfluss über die einzelnen kortikalen Schichten gewährleistet (Abbildung 3,4). Ein zentrales Element kortikaler Signalverarbeitung stellen die exzitatorischen Neurone der Schicht 4 dar, über deren axonale Projektion die Information selektiv in obere Kortexschichten und innerhalb der kortikalen Kolumne verteilt wird. Über das weitreichende System horizontaler Axonkollateralen der Pyramidenneurone der Schichten 2/3 und 5 wird die Ausbreitung der sensorischen Information über kortikale Kolumnen hinweg und über ihre callosale Projektion zur Gegenseite ein Abgleich beider kortikaler Hemisphären sichergestellt.

\section{Inhibitorische Interneurone}

In dem oben beschriebenen Modell zum Signalfluss in einer kortikalen Kolumne sind GABAerge Interneurone bewusst ausgeklammert worden und werden hier gesondert betrachtet. Obwohl diese Neurone nur ca. 10-15\% der gesamten Neuronenpopulation des Neokortex repräsentieren und verschiedene Typen (Klassen) dieser Neurone schon sehr lange bekannt sind, ist ihre Funktion und damit ihre Rolle innerhalb des kolumnären Netzwerks im Gegensatz zum Hippokampus bis heute relativ unklar. Die hohe Zielspezifität ihrer Axone an verschiedenen Kompartimenten des Zielneurons führt zu einer deutlich unterschiedlichen selektiven Art der Inhibition innerhalb des kortikalen Netzwerks, die weit über den Mechanismus der Feed-forward- und Feed-back-Inhibition hinausgeht (Kawaguchi und Kubota 1997, 1998; Buhl et al. 1997; Tamás et al. 1997, 1998; Galarreta und Hestrin 1999, 2001; Markram et al. 1998; Reyes et al. 1998; Gibson et al. 1999; Zilberter et al. 1999;
Gupta et al. 2000; Krimer und GoldmanRakic 2001; Kaiser et al. 2004; Karube et al. 2004). Es gilt heute als gesichert, dass GABAerge Interneurone via chemischer und elektrischer Synapsen untereinander neuronale Ensembles bilden, die für die Synchronisation und Aufrechterhaltung oszillatorischer Aktivität verantwortlich sind (Galarreta und Hestrin 1999, 2001; Gibson et al. 1999; Támas et al. 2000). Synaptische Verbindungen zwischen exzitatorischen und inhibitorischen Neuronen sind rekurrent und scheinen eine wichtige Rolle bei der Stabilisierung des kortikalen Netzwerks zu spielen, indem sie das Auftreten von ,run away“ Exzitation und paroxysmaler neuronaler Aktivität unterdrücken, wie sie in unkontrollierten exzitatorischen Netzwerken vorkommen kann. Darüber hinaus unterliegen exzitatorische Synapsen einer viel stärkeren Depression als inhibitorische Synapsen, selbst während Perioden anhaltender neuronaler Aktivität. Sie besitzen daher auch einen intrinsischen Inhibitionsmechanismus, der zur Stabilität neuronaler Netzwerke beiträgt (Galarreta und Hestrin 1998). Im Gegensatz dazu wird durch die verschiedenen Typen GABAerger Interneurone ein hoher Grad der Flexibilität bei der Regulation neuronaler Aktivität erreicht.

\section{Synaptische Effizienz und Verlässlichkeit synaptischer Übertragung}

Für ein genaues Verständnis neuronaler Verrechnungsprozesse in einer kortikalen Kolumne ist aber nicht nur die Konnektivität zwischen Neuronen sondern auch die Stärke oder Effizienz der verschiedenen synaptischen Verbindungen von entscheidender Bedeutung. Sie wird durch drei voneinander unabhängige Parameter bestimmt: (1) der Anzahl der Freisetzungsstellen des Neurotransmitters $\boldsymbol{n}$, (2) der Wahrscheinlichkeit der Transmitterfreisetzung aus synaptischen Vesikeln $\boldsymbol{P}_{r}$ pro Verbindung, und (3) der quantalen Amplitude $\boldsymbol{q}$, d.h. der Signalamplitude pro synaptischen Kontakt. Synaptische Effizienz ist somit das Produkt dieser drei Parameter $\mathbf{E}=\boldsymbol{n} \cdot \boldsymbol{q} \cdot \boldsymbol{P}_{r}$ und wird durch die Amplitude postsynaptischer Potentiale (EPSP oder IPSP, je nachdem, ob die Verbindung exzitatorisch oder inhibitorisch ist) oder Ströme (EPSC oder IPSC) ausgedrückt.

Individuelle synaptische Verbindungen des Neokortex zeigen große Unterschiede bezüglich ihrer synaptischen Effizienz (Feldmeyer und Sakmann 2000; zusammengefasst durch Thomson und Bannister 2003). Synaptische Verbindungen zwischen exzitato- rischen Sternzellen der kortikalen Schicht 4 zeichnen sich durch eine hohe EPSP Amplitude $(1.6 \mathrm{mV})$ bei niederfrequenter Stimulation aus (Feldmeyer et al. 1999), während andere kortikale Verbindungen, z.B. solche zwischen Pyramidenneuronen der Schicht 6 und Sternzellen der Schicht 4, eine 16-fach niedrigere EPSP Amplitude $(0.1 \mathrm{mV})$ aufweisen (Stratford et al. 1996).

Die große Mehrzahl exzitatorischer Verbindungen des Neokortex ist jedoch durch eine relativ hohe Wahrscheinlichkeit der Transmitterfreisetzung charakterisiert. Translaminäre synaptische Verbindungen zwischen Sternzellen und Schicht 2/3-Pyramidenneuronen im Barrel-Kortex haben hohe $P$ Werte (0.8). Ähnlich verhält es sich bei intralaminären Verbindungen zwischen Pyramidenneuronen der Schicht $2 / 3$ und zwischen Sternzellen der Schicht 4 (Feldmeyer et al. 1999). Demzufolge besitzen eine Reihe von intrakortikalen synaptischen Verbindungen eine $P_{r}$, die fast so hoch ist wie diejenige der sehr verlässlichen thalamokortikalen Verbindungen (Gil et al. 1999). Daneben existieren jedoch auch Verbindungen mit eher niedrigen $P_{r}$, z.B. Verbindungen zwischen Schicht 6-Pyramidenneuronen und Schicht 4-Neuronen des visuellen Kortex (0.37-0.53; Stratford et al. 1996) und einer Subpopulation synaptisch gekoppelter Schicht 2/3-Pyramidenneuronen im auditorischen Kortex (0.13, Atzori et al. 2001). Diese Befunde sprechen für eine hohe Effizienz und Verlässlichkeit der exzitatorischen synaptischen Transmission neokortikaler (intrinsischer) Verbindungen und wurden bis dato für intrinsische kortikale Verbindungen kontrovers diskutiert (Stevens 1994).

Die Wahrscheinlichkeit der Transmitterfreisetzung und damit die Amplitude der EPSPs bzw. EPSCs hängt entscheidend von der Dynamik, d.h. dem „Funktionszustand“ des präsynaptischen Neurons ab. Die repetitive Stimulation des präsynaptischen Neurons einer exzitatorischen Verbindung des Neokortex führt im nachgeschalteten postsynaptischen Neuron in der Regel zu synaptischer Depression (engl. Paired Pulse Depression), während inhibitorische Verbindungen oft eine synaptische Faszilitierung (engl. Paired Pulse Fascilition) zeigen. Das frequenzabhängige Verhalten einer Synapse, ihre Kurzzeit-Plastizität, ist oft zielneuronspezifisch (Stratford et al. 1996; Reyes und Sakmann 1999; Reyes et al. 1998; Galaretta und Hestrin 1998; Makram et al. 1998; Thomson und Bannister 1998; Varela et al. 1999; Gupta et al. 2000; Krimer und Goldman-Rakic 2001; Feldmeyer et al. 2002; Kaiser et al. 2004); regionenspezifisch (Atz- 
ori et al. 2001), wird über die postnatale Entwicklung reguliert (Reyes und Sakmann 1999) und zeigt einen hohen Grad der Variabilität bezüglich der Spezifität der Verbindung (Tsodyks und Markram 1997).

Sensorische Deprivation, z.B. das Trimmen der Schnurrhaare, scheint nach neueren Untersuchungen nicht nur einen strukturellen Effekt auf die axonale und dendritische Morphologie intra- bzw. transkolumnärer Verbindungen zu besitzen, sondern auch einen signifikanten funktionellen Einfluss auszuüben, z.B. auf die Kurzzeitdynamik der synaptischen Transmission (Finnerty et al. 1999; Keller und Carlson 1999; Bender et al. 2003; Maravall et al. 2004, Petersen et al. 2004).

Die Effizienz der Signalübertragung sensorischer Information ist maximal für eine bestimmte Frequenz der Aktivierung an einer Synapse. Neokortikale Synapsen, die neuronale Aktivität herunterfahren, tragen wesentlich zur räumlichen Verarbeitung von Signalen während niederfrequenter Stimulation bei, z.B. während spontaner Entladung. Synapsen mit faszilitierenden Eigenschaften scheinen dagegen erst bei hochfrequenter Stimulation $(9-70 \mathrm{~Hz})$ optimal zu operieren und könnten daher eher bei evozierter Aktivität kortikaler Neurone rekrutiert werden (Fuhrmann et al. 2002). Es ist jedoch weiterhin unklar, ob spontane Aktivität in vivo (im wachen Tier) und evozierte Aktivität durch sensorische Stimulation als gleichwertig angesehen werden können (Moore und Nelson 1998, Zhu und Connors 1999; Brecht und Sakmann 2003).

\section{Quantale Eigenschaften der synaptischen Übertragung}

Ein synaptischer Kontakt besteht aus morphologischer Sicht aus einer präsynaptischen Neurotransmitter-Freisetzungsstelle, an der ein „Pool“ synaptischer Vesikel lokalisiert ist, einer postsynaptischen Dichte, an der je nach Synapsentyp verschiedene NeurotransmitterRezeptoren zu finden sind und einem synaptischen Spalt zwischen prä- und postsynaptischer Membran. Es ist bis heute jedoch unklar, wie synaptische Übertragung in einer definierten Verbindung abläuft. Ein bestimmender Faktor ist hier die Anzahl an Neurotransmitter-Freisetzungsstellen. Es ist aber nicht eindeutig geklärt, ob alle morphologisch identifizierten synaptischen Kontakte einer Verbindung auch funktionell sind (Phänomen der silent synapses), und ob es pro Kontakt mehr als nur eine Neurotransmitter-Freisetzungsstelle gibt. So gesehen bestimmt die Anzahl an Freisetzungsstellen die Eigenschaften synaptischer Übertragung, z.B. ob uni- bzw. multivesikulärer und/oder uni- bzw. multiquantale Freisetzung des Neurotransmitters aus synaptischen Vesikeln vorliegt. Diese Problematik wurde bisher nur in wenigen Studien thematisiert (Korn et al. 1981; Gulyas et al. 1993; Silver et al. 2003). Trotz großer Unterschiede in Bezug auf die Anzahl identifizierter synaptischer Kontakte der bisher untersuchten synaptischen Verbindungen (zwischen InterneuronMauthnerzelle im Goldfisch, hippokampaler CA3 PyramidenzelleInterneuron und der kortikalen Sternzelle-Schicht 2/3-Pyramidenzelle der Ratte) zeigte sich, dass die Anzahl funktionell bestimmter Transmitter-Freisetzungsstellen der Anzahl elektronenmikroskopisch identifizierter synaptischer Kontakte entsprach. Diese Ergebnisse sprechen eindeutig für funktionelle Kontakte mit nur einer Freisetzungsstelle.

Bis heute wird kontrovers diskutiert, ob an zentralen Synapsen uni- oder multivesikuläre Freisetzung an aktiven Zonen stattfindet, d.h. ob nur ein oder mehrere Vesikel an der Freisetzung des Neurotransmitters pro Aktivierung beteiligt sind. Die Hypothese one site one vesicle resultiert aus klassischen Studien, in denen die quantale Freisetzung, bestimmt durch statistische Analyse synaptischer Potentiale, mit der Anzahl morphologisch identifizierter Freisetzungs- stellen nahezu übereinstimmt (Korn et al. 1981; Redman 1990; Gulyas et al. 1993; Korn et al. 1994). Auf der anderen Seite liegen immer mehr ultrastrukturelle Daten vor, die belegen, dass die Zahl sogenannter „docked vesicles “ an zentralen Synapsen unterschiedlich hoch ist, aber definitiv mehr als nur ein Vesikel an die präsynaptische Membran gedockt ist, welches für eine multivesikuläre Freisetzung spricht (Harris und Sultan 1995; Schikorski und Stevens 1997; Xu-Friedmann et al. 2001; Millar et al. 2002). Diese morphologischen Befunde werden durch physiologische Studien unterstützt, die ebenfalls eine multivesikuläre Transmitterfreisetzung an zentralen Synapsen postulieren (Wadiche und Jahr 2001; Oertner et al. 2002). Der Vergleich beider Hypothesen lässt den Schluss zu, dass $a$ priori wahrscheinlich beide Mechanismen an zentralen Synapsen vorkommen. Für die Verbindung zwischen exzitatorischen Sternzellen und Schicht 2/3-Pyramidenzellen haben wir zeigen können, dass pro Aktivierung eines synaptischen Kontakts dieser Verbindung nur ein Transmitter-Vesikel freigesetzt wird (Silver et al. 2003). Demnach folgt ein synaptischer Kontakt dem Alles-oder-Nichts-Prinzip: Entweder wird Transmitter freigesetzt, daraus resultiert ein postsynaptisches Signal, oder es kommt zu keiner Antwort. Die Fluktuation der Signalamplitude (EPSP) ist also allein auf die unterschiedliche Zahl aktiver Kontakte pro Verbindung zurückzuführen. Ob dies jedoch ein allgemein gültiges Prinzip für andere zentrale oder kortikale Synapsen ist, muss in Folgearbeiten noch eindeutiger geklärt werden.

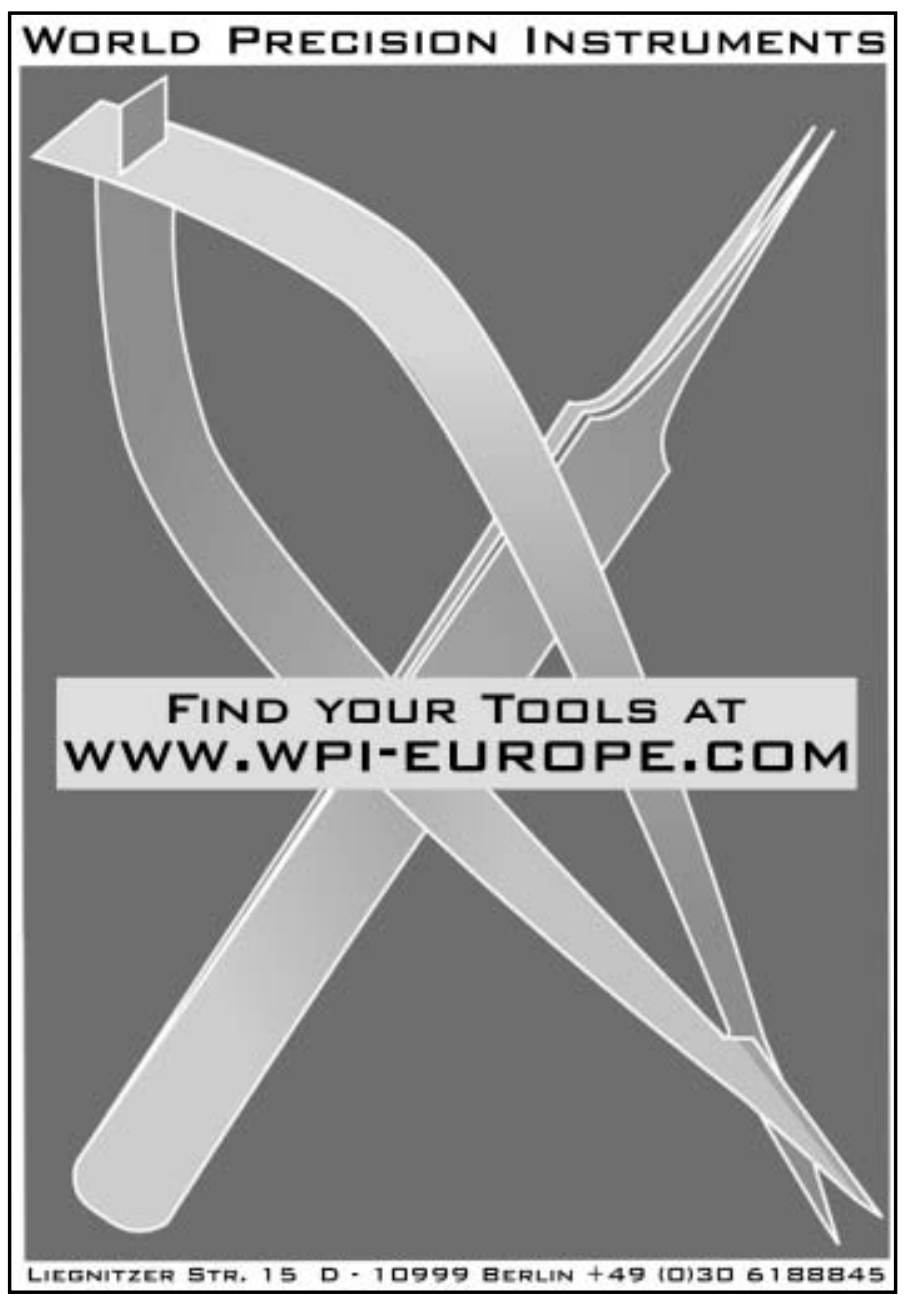




\section{Literatur}

Atzori, M., Lei, S., Evans, D.I.P., Kanold, P.O., Phillips-Tansey, E., McIntyre, O. und McBain, C.F. (2001): Differential synaptic processing separates stationary from transient inputs to the auditory cortex. Nature Neuroscience 4: 12301237.

Braitenberg, V. und Schüz, A. (1998): Anatomy of the Cortex: Statistics and geometry. Berlin: Springer Verlag. Ed. 2.

Buhl, E.H., Tamás, G., Szilágyi, T., Stricker, C., Paulsen, O. und Somogyi, P. (1997): Effect, number and location of synapses made by single pyramidal cells onto aspiny interneurones of cat visual cortex. Journal of Physiology 500: 689-713.

Deuchars, J., West, D.C. und Thomson, A.M (1994): Relationships between morphology and physiology of pyramid-pyramid single axon connections in rat neocortex in vitro. Journal of Physiology 478: 423-435.

Feldmeyer, D., Egger, V., Lübke, J. und Sakmann, B. (1999): Reliable synaptic connections between pairs of excitatory layer 4 neurones within a single 'barrel' of developing rat somatosensory cortex. Journal of Physiology 521: 169-190.

Feldmeyer, D. und Sakmann, B. (2000): Synaptic efficacy and reliability of excitatory connections between the principal neurones of the input (layer 4 ) and output layer (layer 5) of the neocortex. Journal of Physiology 525: 31-39.

Feldmeyer, D., Lübke, J., Silver, R.A. und Sakmann, B. (2002): Synaptic connections between layer 4 spiny neurones and layer $2 / 3$ pyramidal cells in rat barrel cortex: physiology and anatomy of interlaminar signaling within a cortical column. Journal of Physiology 538: 803-822.

Galarreta, M. und Hestrin, S. (1999): A network of fast-spiking cells in the neocortex connected by electrical synapses. Nature 402: 72-75.

Gulyás, A.I., Miles, R., Sik, A., Tóth, K., Tamamaki, N. und Freund T.F. (1993): Hippocampal pyramidal cells excite inhibitory neurons through a single release site. Nature 366: 683-687.

Hubel, D.H. und Wiesel, T.N. (1962): Receptive fields, binocular interaction and functional architecture in the cat's visual cortex. Journal of Physiology 160: 106-154.

Kawaguchi, Y. und Kubota, Y. (1997): GABAergic cell subtypes and their synaptic connections in rat frontal cortex. Cerebral Cortex 7: 476-486.

Korn, H., Friller, A., Mallet, A., Legrende, P. und Faber, D.S. (1981): Fluctuating response at a central synapse: $\mathrm{n}$ of binomial fit predicts number of presynaptic boutons. Science 213: 898901.

Korn, H., Sur, C., Stephane, C., Legrende, P. und Faber, D.S. (1994): The one-vesicle hypothesis and multivesicular release. In: Stärnje, L., Greengard, P., Grillne, S., Hökfeldt, T. und Ottoson, D. (Hrsg.) Molecular and cellular mechanisms of neurotransmitter release. New York: Raven Press.

Lübke, J., Egger, V., Sakmann, B. und Feldmeyer, D. (2000): Columnar organization of dendrites and axons of single and synaptically coupled neurons in layer 4 of the rat barrel cortex. Journal of Neuroscience 20: 5300-5311.
Lübke, J., Roth, A., Feldmeyer, D. und Sakmann, B. (2003): Morphometric analysis of the columnar innervation domain of neurons connecting layer 4 and layer $2 / 3$ of juvenile rat barrel cortex. Cerebral Cortex 13: 1051-1063.

Markram H., Lübke J., Frotscher M., Roth A. und Sakmann B. (1997a): Physiology and anatomy of synaptic connections between thick tufted pyramidal neurones in the developing rat neocortex. Journal of Physiology 500: 409-440.

Mountcastle, V.B. (1957): Modality and topographic properties of single neurons of cat's somatic sensory cortex. Journal of Neurophysiology 20: 408-434

Petersen, C.C., Brecht, M., Hahn, T.T. und Sakmann, B. (2004): Synaptic changes in layer $2 / 3$ underlying map plasticity of developing barrel cortex. Science 304: 739-742.

Schikorski, T. und Stevens, C.F. (2001): Morphological correlates of functionally defined synaptic vesicle populations. Nature Neuroscience 4: 391-395.

Silver, R.A., Lübke, J., Sakmann, B. und Feldmeyer, D. (2003): Uniquantal transmission at excitatory synaptic contacts in barrel cortex. Science 302: 1981-1984.

Támas, G., Somogyi, P. und Buhl, E.H. (1998): Differentially interconnected networks of GABAergic interneurons in the visual cortex of the cat. Journal of Neuroscience 18: 4255-4270.

Thomson, A.M., Deuchars, J. und West, D.C. (1993): Single axon excitatory postsynaptic potentials in neocortical interneurons exhibit pronounced paired pulse facilitation. Neuroscience 54: 347-360.

Thomson, A.M., West, D.C., Wang, Y. und Bannister, A.P. (2002): Synaptic connections and small circuits involving excitatory and inhibitory neurons in layers 2-5 of adult rat and cat neocortex: triple intracellular recordings and biocytin labelling in vitro. Cerebral Cortex 12: 936-953.

Wadiche, J.I. und Jahr, C.E. (2001): Multivesicular release at climbing fiber-Purkinje cell synapses. Neuron 32: 301-313.

Eine ausführliche Literaturliste kann bei den Autoren angefordert werden.

\section{Danksagung}

Wir möchten an dieser Stelle der Deutschen Forschungsgemeinschaft für die kontinuierliche finanzielle Unterstützung in Form von Einzel bzw. SFB-Anträgen danken, die wesentlich zum Erfolg unserer Forschungsarbeiten beigetragen haben. Unser besonderer Dank gilt Prof. Dr. Bert Sakmann und Prof. Dr. Michael Frotscher für die stetige wissenschaftliche Unterstützung unserer Forschungsvorhaben.

\section{Kurzbiographien}

Joachim Lübke: geboren 1956; 1974-1977: Ausbildung zum biologisch-technischen Assistenten. 1981-1982: Begabten-Abitur am Abendgymnasium in Göttingen. 1982-1987:
Studium der Biologie in Göttingen. 19871991: Diplom- und Doktorarbeit am MaxPlanck-Institut für biophysikalische Chemie, Abt. Neurobiologie (Prof. Dr. O.- D. Creutzfeldt). 1991-1993: Postdoctoral Fellow (Stipendiat der Royal Society of Science) am Department of Human Anatomy (Prof. Dr. Ray Guillery) der University of Oxford. 19931995: „Von Helmholtz“-Stipendiat des BMBF am Institut für Anatomie der Universität Freiburg (Prof. Dr. Michael Frotscher). 1997: Wolfgang-Bargmann-Preis der Anatomischen Gesellschaft. 1999: Habilitation im Fach Anatomie an der Universität Freiburg. 2000: Ernennung zum Hochschuldozenten C2. 2003: Berufung auf die Abteilungsleiterstelle Zelluläre Neurobiologie am Institut für Medizin, Forschungszentrum Jülich GmbH.

Dirk Feldmeyer: geboren 1960; 1979-1983: Studium der Biologie, Germanistik und Pädagogik an der Ruhr-Universität Bochum. 1984 1987: Doktorarbeit am Institut für Zellphysiologie (Prof. Dr. H. C. Lüttgau). 1990-1995: Postdoctoral Fellow (DFG, Welcome Trust) am Department of Pharmacology, UCL London (Prof. S.G. Cull-Candy).1994: Forschungsaufenthalt (Stipendiat des British Council und des Japanese Ministry of Education) am Brain Research Institute in Tokyo (Prof. T. Takahashi). 1995: Berufung auf eine Arbeitsgruppenleiterstelle an das Max-Planck-Institut für medizinische Forschung, Abt. Zellphysiologie in Heidelberg (Direktor: Prof. Dr. med. B. Sakmann). 2000: Habilitation und Ernennung zum Hochschuldozenten für das Fach Physiologie an der Universität Heidelberg. 2001: Berufung zum Editor des Journals of Physiology (London). Ab August 2004 Berufung als Abteilungsleiter Zelluläre Neurobiologie am Institut für Medizin, Forschungszentrum Jülich GmbH.

\section{Korrespondenzadressen}

\section{Joachim Lübke}

Institut für Medizin

Abteilung Zelluläre Neurobiologie

Forschungszentrum Jülich GmbH

D-52425 Jülich

Tel.: ++ $49(0) 2461612288$

Fax: ++49(0) 2461612820

e-mail: j.luebke@fz-juelich.de

\section{Dirk Feldmeyer}

Institut für Medizin

Abteilung Zelluläre Neurobiologie

Forschungszentrum Jülich GmbH

D-52425 Jülich

Tel.: ++49(0) 2461615914

Fax: ++49 (0) 2461612820

e-mail:d.feldmeyer@fz-juelich.de 